\title{
Information und Kommunikation 2005: Ein Lagebericht und einige Zukunftsperspektiven
}

\author{
Hans-Werner Bierhoff, Joachim Funke, Ulf-Dietrich Reips \\ und Erich Weichselgartner
}

\begin{abstract}
Mit dem vorliegenden Beitrag wollen die Mitglieder der föderativen Kommission „Information und Kommunikation“ (IuK) einen Bericht zur aktuellen Lage und zu möglichen Perspektiven geben. Unter dem Titel „Information und Kommunikation" werden hier die strategisch relevanten Implikationen von Intranet und Internet behandelt, die für die Wissenschaften zu einer tiefgreifenden Revolution geführt haben. Waren noch vor rund 20 Jahren die wichtigsten Hilfsmittel von Wissenschaftlern Karteikästen, Schreibmaschinen, Sekretariate, Kopierer, Sonderdruckanforderungen und Fernleihbestellungen, hat sich diese Situation durch elektronische Möglichkeiten zur Information und Kommunikation radikal geändert. Heute geht es darum, mittels Computern aus einer schier überbordenden Menge an Informationsangeboten die relevanten, wichtigen und hochwertigen auszuwählen und bei der meist eigenhändigen Produktion wissenschaftlicher Texte am Computer zu nutzen.
\end{abstract}

Noch nie waren so viele Informationen verfügbar wie heute, aber noch nie war zugleich das Bedürfnis nach qualifizierter Reduktion des Überangebots so hoch. Auch die Möglichkeiten zur Publikation von Information sind durch das Internet auf völlig neue Beine gestellt worden: heute braucht man nicht zwingend einen Verleger, um wissenschaftliche Information zu distribuieren, sondern kann dies über einen Server kostengünstig und mit hoher Effizienz in die eigene Hand nehmen.

In diesem Beitrag wollen wir einige der aktuellen Entwicklungen aufgreifen und kommentieren sowie Vorschläge machen, wie unser Fach und wie die DGPs auf diese zum Teil stürmischen Entwicklungen reagieren kann. Wir beginnen mit einer kurzen Darstellung, wie die veränderten Strukturen im Bereich IuK nicht nur die Psychologie, sondern alle Bereiche der Wissenschaft grundlegend verändert haben. Es folgt der Versuch, den Status Quo zu skizzieren. Hierzu wollen wir verschiedene „Spieler“ auf dem IuK-Markt vorstellen, die dort gehandelten Produkte darstellen, die zu ihrer Bewertung herangezogenen Kriterien betrachten und schließlich Empfehlungen und Lösungsvorschläge behandeln.

Der vorliegende Beitrag gibt Bewertungen und Einschätzungen der Kommissionsmitglieder wieder, die nicht denjenigen des BDPPräsidiums bzw. des DGPs-Vorstands entsprechen müssen. Die Erstfassung dieses Beitrags erfolgte 2004.

\author{
Die Open-Access-Initiative (OAI) \\ und weitere Änderungen \\ in der luK-Landschaft
}

\begin{abstract}
Das, was heute als Open-Access-Initiative (OAI) bezeichnet wird, hat unter anderem unter dem Begriff „Open-Archive-Initiative“ eine lange Vorgeschichte. Insbesondere ist in diesem Zusammenhang Steven Harnad zu erwähnen, der seit Ende der 80er Jahre aktiv für „Scholarly Skywriting" (elektronischer Austausch von Informationen in Forschergruppen während aller Phasen der Publikationsgenese von anfänglichen Planungen bis hin zum Versenden von Vorabdrucken) eintritt (Harnad, 1990). Er hat die Möglichkeiten des Internet für die Weiterentwicklung wissenschaftlicher Kommunikation in zahlreichen Beiträgen hervorgehoben und dafür plädiert, dass Wissenschaftler sich nicht mehr zwischengeschalteten Verlagen bei der Distribution ihrer Forschungsergebnisse bedienen, sondern eine online-gestützte Form direkter Kommunikation praktizieren sollten. Dabei war für ihn von Anfang an klar, dass das Erfolgsprinzip „Peer Reviewing“ auch in neuen Formen von Online-Journals erhalten bleiben musste. Dass Verleger mit dieser Strategie nicht glücklich waren, kann man sich leicht vorstellen.
\end{abstract}

Wichtige historische Ereignisse waren 1971 der Start des „Projekts Gutenberg“ durch Michael Hart, 1991 die Einrichtung des ersten „Preprint-Servers“ durch den Physiker Paul Ginsparg, 1995 die Gründung der IuK-Initiative der wissenschaftlichen Fachgesellschaften in Deutschland, bei der die Deutsche Gesellschaft für Psychologie ebenfalls vertreten ist, sowie die Budapest Open Access Initiative (BOAI), die durch den ehemaligen Finanzspekulanten George Soros massiv unterstützt wurde und im Jahre 2002 den Gedanken formuliert hat, dass Wissenschaftler ihre Erkenntnisse unbeschränkt und frei online zugänglich machen sollten. Die BOAI hat nicht nur die Forderung nach Open-Access-Journals gestellt, sondern auch für jeden einzelnen Wissenschaftler die Forderung aufgestellt, seine eigenen Artikel online zugänglich zu machen und damit zum ,Self-Archiving“ beizutragen.

Die Budapester Initiative ist ergänzt worden durch die „Berliner Erklärung über offenen Zugang zu wissenschaftlichem Wissen“, die von den Präsidenten der FraunhoferGesellschaft, der Max-Planck-Gesellschaft, der Deutschen 
Forschungsgemeinschaft, der Hochschul-Rektoren-Konferenz, dem Wissenschaftsrat und weiteren großen Forschungseinrichtungen in der Bundesrepublik unterzeichnet wurde.

In ähnliche Richtung gehen auch die Empfehlung der Hochschul-Rektoren-Konferenz, ,Zur Neuausrichtung des Informations- und Publikationssystems der Deutschen Hochschulen“ vom 5. November 2002, die „Empfehlung zur digitalen Informationsversorgung durch Hochschulbibliotheken“, die der Wissenschaftsrat am 13. Juli 2001 verabschiedet hat, sowie die Stellungnahme des Deutschen Bibliotheksverbandes zum Strategiekonzept ,Zukunft der wissenschaftlichen und technischen Information“ vom 27. Juli 2001.

Der Grundgedanke von Open-Access besteht darin, dass wissenschaftliche Literatur digital online kostenfrei und frei von sonstigen Restriktionen zugänglich gemacht wird (keine Copyright- und Lizenz-Restriktionen). OpenAccess verträgt sich mit dem Verfahren des Peer Reviewing, verhindert nicht die Erzeugung von Druckwerken und erlaubt natürlich auch Indexierung und andere Unterstützungsmöglichkeiten, wie sie auch für konventionelle Fachliteratur verfügbar sind. Da Wissenschaftler normalerweise ihre Forschungsergebnisse publizieren, ohne dafür monetäre Vergütung zu erhalten, wäre durch dieses Verfahren ausschließlich der Zwischenhändler (= Verlag) benachteiligt und müsste Mehrwertdienste entwickeln, die entsprechende Preise rechtfertigen würden. Da natürlich der freie Zugang zu wissenschaftlicher Literatur nicht heißt, dass diese kostenfrei produziert und vertrieben werden kann, sind Geschäftsmodelle im Gespräch, bei denen zum Beispiel der Produzent für die Publikation seiner Arbeit eine pauschale Gebühr entrichtet und damit die Konsumenten von der Zahlungspflicht entbindet. Diese Umkehrung der gegenwärtigen Verhältnisse (gegenwärtig zahlt der Autor nichts, ${ }^{1}$ die Leser alles) würde natürlich auch eine Umkehrung der Investition öffentlicher Mittel bedeuten: Anstelle von Millioneninvestitionen für die Abonnements wissenschaftlicher Zeitschriften könnte dieses Geld für die Produktion und Bereitstellung wissenschaftlicher Artikel investiert werden, da dann eben keine Kosten für den Bezug mehr bestünden. Aber von diesem Denkmodell ist einstweilen noch nicht viel zu sehen. Um es konkret umzusetzen, sind betriebswirtschaftliche Kostenrechnungen erforderlich. Steven Harnad rechnet vor, dass die wissenschaftlichen Einrichtungen 90\% ihrer Bibliotheksetats einsparen könnten.

Die Initiative „Public Library of Science“ (PLoS) ist ebenfalls eine wichtige Entwicklung, da in diesem Modell seit 2003 eine erste elektronische Zeitschrift „PLoS Biolo-

\footnotetext{
${ }^{1}$ Dies ist nicht ganz richtig: Schon heute gibt es Zeitschriften, bei denen Autoren eigene Zahlungen leisten müssen. Dies gilt in verstärktem Maße für die Publikation von Diplomarbeiten, Dissertationen und Habilitationsschriften. Weiterhin ist zu bedenken, dass die Institutionen, an denen die Autoren beschäftigt sind, zumindest einen Teil der Forschung finanzieren. Über diese Forschung wird dann in Zeitschriften berichtet.
}

gy“ mit großem Erfolg platziert werden konnte; ,PLoS Medicine“ ist in 2004 nachgefolgt. Ob es eine „PLoS Psychology" geben wird, ist nicht bekannt, aber laut „Directory of Open Access Journals“ gibt es schon 21 Open-Access Psychologie-Zeitschriften. Darüber hinaus werden ältere Jahrgänge von Zeitschriften zunehmend frei ins Netz gestellt, z. B. das Journal of Applied Behavior Analysis und das Journal of the Experimental Analysis of Behavior mit zweijährigem Verzug.

\section{Internet-basierte Forschung}

In der Internet-Forschung (Internet Science) wird die Psychologie Internet-bezogenen Verhaltens (z. B. Joinson, 2003) von der Nutzung des Internet für die Durchführung von Forschung (deshalb „Internet-basierte“ Forschung) unterschieden (Reips, 2003, in press). Ersteres ist Gegenstand vor allem der Medienpsychologie, letzteres der Methodik. Wir berichten hier über den Stand der Internetbasierten psychologischen Forschung.

Die Entwicklung und Nutzung der Internet-basierten psychologischen Forschung hat in den Jahren seit dem Aufkommen des Internet exponentiell zugenommen. Dies ist nicht verwunderlich, denn sie wirbt mit einer ansehnlichen Liste praktischer und methodischer Argumente für ihren Einsatz (siehe z. B. Birnbaum, 2004; Bosnjak, 2003; Buchanan, 2001, 2002; Reips, 2000, 2002 b). Diese Forschungsmethodik versucht die Vorteile computer-basierter Erhebungen (z.B. Automatisierung, Standardisierung) mit denen des weltweiten Netzes (z. B. Erreichbarkeit) zu verbinden. Einige der Hauptvorteile sind: a) eine große Teilnehmerzahl kann sehr schnell untersucht werden; b) es können große heterogene Stichproben und Personen mit seltenen Merkmalen rekrutiert werden; c) die Methode ist kostengünstiger im Hinblick auf Zeit, Räumlichkeiten und Arbeitsaufwand (Birnbaum \& Reips, in press).

Das weltweit erste Experimentallabor im Internet wurde 1995 an der Universität Tübingen eingerichtet und ist inzwischen an der Universität Zürich beheimatet: das Web-Labor für Experimentelle Psychologie (http://www. psychologie.unizh.ch/sowi/Ulf/Lab/WebExpPsyLab. html). Neben den dort aufgelisteten Web-Experimenten kann man sich auf den folgenden Websites einen Eindruck von Internet-basierten Untersuchungen verschaffen:

- Web experiment list: http://genpsylab-wexlist.unizh.ch/

- Web survey list: http://genpsylab-wexlist.unizh.ch/ browse.cfm?action=browse \&modus=survey

- Psychological Research on the Net (John Krantz): http://psych.hanover.edu/research/exponnet.html

- International Personality Item Pool (Lewis R. Goldberg): http://ipip.ori.org/ipip/

- Online Social Psychology Studies (Scott Plous): http:// www.socialpsychology.org/expts.htm 
Auch die DGPs hat sich in ihrer Mitgliederbefragung zu Publikationserfahrungen und Open Access für das Internet-basierte Format entschieden. Über Ergebnisse und Erfahrungen damit wird an dieser Stelle in Kürze berichtet werden.

Es hat sich gezeigt, dass Internet-basierte Forschung in der Regel valide Ergebnisse liefert (Krantz \& Dalal, 2000), sogar bei störanfälligen Maßen wie Reaktionszeiten (Eichstaedt, 2001; Reips, Morger \& Meier, 2001). Insgesamt werden mögliche Nachteile durch die wissenschaftstheoretischen und praktischen Vorteile mehr als ausgeglichen (Buchanan, 2002; Musch \& Reips, 2000; Reips, 2000, 2002 b).

Mit Hilfe von im Internet verfügbaren Werkzeugen in Form so genannter ,Web services“ lässt sich die Durchführung von Internet-basierten Untersuchungen leicht erlernen und handhaben. Hier findet zum Teil eine Integration mit e-Learning statt: Die Werkzeuge können auch gut in der Lehre eingesetzt werden. Beispiele für solche Web services sind:

- SurveyWiz (Michael Birnbaum, einfache Befragungen): http://psych.fullerton.edu/mbirnbaum/programs/ surveyWiz.htm

- Inquiry (FH Ravensburg-Weingarten, Befragungen): http://www.inquiry.de/

- WEXTOR (Generieren, Visualisieren und Verwalten von experimentellen Versuchsdesigns, Erstellen von Webseiten und Versuchssteuerung): http://psych-wextor. unizh.ch/wextor/en/index.php

- Stroop Invaders (Erstellen von Stroop-Experimenten im Spiel-Format): http://www.psychologie.unizh.ch/sowi/ reips/stroop/

- Scientific LogAnalyzer (Auswertung beliebiger Logdateien): http://genpsylab-logcrunsh.unizh.ch/

Die Einführung der Internet-basierten Forschungsmethodik in universitäre Curricula ist dringend geboten, denn im Internet durchgeführte Untersuchungen werden einerseits sehr gerne von Studierenden durchgeführt und zeigen andererseits derzeit eine hohe Rate an methodischen Problemen und Konfigurationsfehlern (Reips, 2002a). Solche Untersuchungen sind wegen ihres öffentlichen Charakters stets auch ein Ausweis der durchführenden Institution.

Die Integration von Online-Kommunikation mit Internet-basierter Forschung und dem Publizieren und SelbstArchivieren in Open Access-Zeitschriften und -Archiven bietet die Chance, insgesamt in einen fruchtbareren Forschungskreislauf einzutreten. Beispielsweise können Internet-basierte Experimente direkt mit dem Methodenteil im dazugehörigen Artikel in einer Online-Zeitschrift verlinkt werden, so dass die Leser selbst die Erfahrung einer Versuchsperson nacherleben können (Reips, 2000, 2003). Als Forscher können sie dann die Materialien kopieren und verändern, um eine sich anschließende Forschungsfrage zu untersuchen. Es ist offensichtlich, dass Internetbasierte Datenarchivierung und e-Learning ebenfalls sinnvoll von diesem Internet-gestützten Forschungsprozess profitieren können.

\section{Informationssuche und Informationsauswertung}

Durch die stürmische Entwicklung des Internet haben sich völlig neue Formen der Informationssuche und Informationsauswertung ergeben. Ein Beispiel ist der Erfolg von Google. Diese Internet-Suchmaschine ermöglicht es, Namen, Zitate und Quellen aufzufinden, die aus der Presse, der Kultur, der Wissenschaft und anderen Bereichen entnommen sind. Die Vielfalt der auf diesem Weg zugänglichen Information hat in den letzten fünf Jahren dramatisch zugenommen.

Allerdings lassen sich qualitative Unterschiede zwischen verschiedenen Bereichen feststellen. Während kulturelles Wissen in vielfältiger Weise im Internet zur Verfügung gestellt wird, sind wissenschaftliche Erkenntnisse, die auf aktueller Forschung beruhen, in der Tendenz weniger erschöpfend vorhanden. Daher stellt sich die Frage, wie die Verfügbarkeit qualifizierter wissenschaftlicher Informationen im Internet verbessert werden kann. Diese Verfügbarkeit muss aber nicht immer Zugänglichkeit zu Informationen implizieren. Zum Beispiel sind im „Ex Libris Link Resolver“ mehr als 1.200.000 Volltexte verfügbar, ohne dass sie jedermann zugänglich wären. Es gibt viele digitale wissenschaftliche Informationen, aber es gibt Probleme damit. Information, die vorhanden ist, wird zurückgehalten. Dadurch kann bei einer Google-Suche der Eindruck entstehen, man würde erfolgreich Fachinformation beschaffen, obwohl das ein nicht zutreffender Eindruck ist. Die APA (American Psychological Association) hält umfangreiche Information vor, aber nur für zahlende Käufer ihrer Informationsdienste.

Bei der Beantwortung dieser Frage lässt sich eine kurzfristige von einer langfristigen Strategie unterscheiden. Kurzfristig geht es um die Frage, ob für psychologische Fachzeitschriften ein pay-per-view-Verfahren bzw. ein free-access-Verfahren eingeführt werden kann. Entsprechende Verhandlungen werden derzeit im Rahmen der von BMBF und DFG geförderten Aktivitäten zur nationalen digitalen Bibliothek (infoconnex, vascoda) mit mäßigem Erfolg geführt. Anders sieht es in Bezug auf ältere Zeitschriftenjahrgänge aus, wie obiges Beispiel der beiden Zeitschriften der Society for the Experimental Analysis of Behavior belegt. Es ist zu vermuten, dass die Verlage geneigter sind, über die Überlassung der Verwertungsrechte alter Jahrgänge von psychologischen Fachzeitschriften und deren angemessene Präsentationsform zu verhandeln.

Langfristig geht es vorrangig um die Frage von Online-Veröffentlichungen im Bereich psychologischer For- 
schung. In diesem Zusammenhang wird auch über die Gründung und Unterstützung elektronischer Fachzeitschriften gesprochen. Solche Entwicklungen von psychologischen Online-Publiziermöglichkeiten könnten unter der Federführung der DGPs stattfinden. Dazu ist neben der Organisation des Review-Systems, der Herausgeberschaft und der technischen Realisierung auch ein Marketing erforderlich, das einer entsprechenden Publikation schon in einer frühen Phase einen angemessenen Impact in der Scientific Community verschafft. Ein mögliches Ziel besteht darin, ein eigenes Internet-basiertes Journal im Auftrag der DGPs anzubieten. Eine andere Möglichkeit wäre, dass die DGPs ein Publikationsdach anbietet, unter dem ihre Mitglieder Zeitschriften im Online-Bereich entwickeln können.

Die Bedeutung von Online-Publikationen wird durch die exorbitanten Kostensteigerungen bei Abonnements von psychologischen Fachzeitschriften hervorgehoben (sog. Zeitschriftenkrise). Für viele Fachbibliotheken stellt der steigende Preis psychologischer Fachzeitschriften ein großes Problem dar. Es ist wünschenswert, dass Lösungen gesucht werden, die eine Verbreitung psychologischer Forschungsergebnisse möglichst umfassend sicherstellen und bestehende Lücken schließen. In diesem Zusammenhang besteht der Wunsch, dass Verlage die Kriterien einer ethischen Unternehmenspolitik erfüllen. Verlage, die von diesen ethischen Kriterien in gravierender Weise abweichen, sollten der Öffentlichkeit bekannt gemacht werden.

Da wissenschaftliche Beiträge in den deutschsprachigen Fachzeitschriften, die vom Marktführer Hogrefe \& Huber Verlagsgruppe betreut werden, erst ab 1999 in der Datenbasis der Verlagsgruppe elektronisch erfasst sind, stellt sich die Frage, ob eine Möglichkeit besteht, auch ältere Jahrgänge elektronisch verfügbar zu machen (notfalls durch Einscannen). Die Erschließung psychologischer Fachzeitschriften im deutschsprachigen Raum vor 1999 erscheint als erstrebenswert. Es wird daran gedacht, dass ein kooperatives Verfahren zwischen der Verlagsgruppe Hogrefe \& Huber und dem ZPID eingeleitet wird, um dieses Ziel zu erreichen. Grundsätzlich sind auch öffentliche Fördermöglichkeiten vorstellbar, die zur digitalen Archivierung älterer Jahrgänge von Fachzeitschriften dienen können. Frühwerke deutscher Psychologie aus dem Beginn der modernen Psychologie sollten für die weltweite Nutzung kostenlos zugänglich gemacht werden.

\section{Open Access: Die beteiligten "Spieler" und ihre Interessenlagen}

Auf dem Publikations-Markt sind mehrere „Spieler“ mit verschiedenen Interessen und in verschiedenen Funktionen tätig. Wir wollen im Einzelnen eingehen auf Autoren, Gutachter, Verlage, Fachgesellschaften, Institute, Bibliotheken, Fachöffentlichkeit und breite Öffentlichkeit.

Für die Autoren wissenschaftlicher Werke gibt es im Wesentlichen zwei Ziele: 1. möchte jeder Autor, dass sein
Werk möglichst breit rezipiert wird, 2. möchte man für die Verbreitung seiner Ideen möglichst keine Kosten übernehmen müssen. Die Visibilität von Artikeln wird häufig an so genannten Impact-Faktoren festgemacht, die die Rezeption wissenschaftlicher Artikel in der Scientific Community abbilden sollen. Für einen Autor geht es also darum, einen möglichst hohen Impact-Faktor bei möglichst geringen eigenen Kosten zu haben. Bei wissenschaftlichen Beiträgen geht es den Autoren in aller Regel nicht um finanziellen Gewinn, zumal sie meist aus öffentlichen Mitteln alimentiert werden und nicht auf derartige Einnahmen angewiesen sind.

Für die Gutachter wissenschaftlicher Werke ist die derzeitige Situation bisher überwiegend so, dass sie ihre Gutachtertätigkeit unentgeltlich (bzw. durch ihre Institutionen gefördert) ausüben. Die Verlage verdienen an dieser Tätigkeit, werden also letztlich durch öffentliche Fördermittel alimentiert.

Die Verlage profitieren in mehrfacher Hinsicht von den neuen Entwicklungen. Zum einen wird das Rohmaterial für wissenschaftliche Zeitschriften wie auch für Bücher heute durch die elektronische Manuskripterfassung weitgehend von den Autoren vorbearbeitet. Der früher übliche Aufwand der Verlage für Satz ist heute auf ein Minimum gefallen, da er weitgehend in der Hand der Autoren liegt. Verlage möchten ihre Produkte (Zeitschriften und Bücher) möglichst zu hohen Preisen am Markt anbieten. Je renommierter eine Zeitschrift ist, umso höhere Preise können gefordert werden.

Die Entwicklung im Buchmarkt hat dazu geführt, dass nicht nur eine Konzentration auf wenige große Verlage stattgefunden hat, sondern zugleich diese großen Verlage nicht mehr an einer breitflächigen Veröffentlichung von Monographien interessiert sind, sondern sich vor allem auf den Markt von Lehrbüchern und populärwissenschaftlich ausgerichteten Werken stützen. Die für ein Fach eigentlich wichtige Unterfütterung des Buchmarktes mit Spezialmonographien ist faktisch zum Erliegen gekommen, wenn man sich die Titelpalette größerer Psychologieverlage ansieht. Häufig werden heute bei Monographien den Autoren Druckkostenzuschüsse in Höhe mehrerer Tausend Euro abverlangt. Auf dem Zeitschriftenmarkt ist die Situation etwas anders gelagert. Hier haben sich durch Konzentrationsprozesse große Anbieter formiert (z. B. Elsevier), die den Bibliotheken ein großes Angebot wissenschaftlicher Zeitschriften als Paket verkaufen. Die Abonnementspreise für Papier- und vor allem für die heute vielfach präferierten elektronischen Zeitschriftenausgaben sind so dramatisch gestiegen, dass die hier sichtbare Preisspirale zu einer verheerenden Situation bei vielen Instituts- und Universitätsbibliotheken geführt hat.

An dieser Stelle kann das Interesse der Bibliotheken erläutert werden. Bibliotheken haben in aller Regel einen Versorgungsauftrag für ihre „Kunden“ (im Wesentlichen die an einer Hochschule tätigen Personen). Durch Zusammenschluss mehrerer Bibliotheken auf Länderebene werden heute Konsortien gebildet, die etwa in Fragen der 
Kontraktierung von Datenbanken und dem Zugang zu Fachinformationen (Zeitschriften etc.) versuchen, kostengünstige Verträge mit den Informationsanbietern abzuschließen. Bibliotheken sehen sich in Zeiten knapper Haushalte kaum noch in der Lage, eine Vollversorgung der verschiedenen Fächer sicher zu stellen.

Damit sind auch zugleich die Probleme für die Fachöffentlichkeit erkennbar. Inwiefern Mitglieder der psychologischen Fachöffentlichkeit Zugang zu allen für sie relevanten Informationen bekommen, ist heute im Wesentlichen eine Frage des Geldbeutels. Im Hinblick auf Eliteförderung ist zu konstatieren, dass der Unterschied zwischen „reichen“ und ,armen“ Universitäten zukünftig vor allem an der Zugänglichkeit von bestimmten Informationen gemessen wird. Darin liegt auch eine Tragik von Dritte-WeltLändern verborgen, die zu bestimmten Informationen kaum noch Zugang bekommen, wenn hier nicht Sonderregelungen vereinbart werden. Noch nie war der Satz „Wissen ist Macht" so klar mit ökonomischen Aspekten verknüpft. Dieser Satz müsste heute lauten: „Information gibt es für den, der zahlen kann" bzw. ,guter, content" ist eine sichere Einnahmequelle“. Es gibt allerdings auch positive Perspektiven, die für Dritte-Welt-Länder mit dem Internet verbunden sind. Denn der Internet-Zugang verschafft ihren Universitäten und Bibliotheken Zugriffsmöglichkeiten auf Informationen, die durch Google und andere Dienste bereitgestellt werden und die ohne das Internet in diesen Ländern nicht verfügbar wären.

Dass mit Fachinformationen auch politische Macht und Einfluss verbunden sein können, demonstriert „,Medline/PubMed“, die eine der größten biomedizinischen Datenbanken darstellt und kostenlos zugänglich ist. Sie wird von der amerikanischen „National Library of Medicine“ und den „National Institutes of Health“ betrieben und sammelt weltweit relevante Einträge. Allerdings bestimmen diese Institutionen auch darüber, was relevant ist. Ähnliche Bestimmungsmacht besitzt das privat betriebene „Institute of Scientific Information“ (ISI), das durch Produkte wie „Current Contents“ und „Social Science Citation Index" vielen Forschenden bekannt ist. Hier als Zeitschrift nicht aufgenommen zu werden, bedeutet eine erhebliche Benachteiligung im wissenschaftlichen Wettbewerb um hohe Visibilität. Mit der Festlegung von Kriterien wie dem Impact-Faktor werden Standards definiert, denen man kaum noch entkommen kann.

Hier ist natürlich auch die breite Öffentlichkeit angesprochen, die an seriöser Information über Psychologie interessiert ist. Frei verfügbare, kompetente Fachinformation ist im Dschungel vielfältigster Angebote kaum zu erkennen, zumal die Kriterien für seriöse und unseriöse Informationen für Außenstehende nicht einfach zu erkennen sind. Hier hat die IuK-Kommission z. B. eine entsprechende Kriterienliste für elektronische Medien veröffentlicht (Funke, Stumpf, Weichselgartner \& Wilkening, 2003), die zur Qualitätssicherung beitragen soll. Dass eine breite Öffentlichkeit trotz Internet oft noch immer nicht an kostenfreie Fachinformation kommt, mutet paradox an angesichts der Tatsache, dass Forschung zu großen Teilen aus Mitteln der Steuerzahlenden finanziert wird.
Ein letztes Wort betrifft die Fachgesellschaften als „Spieler“ auf diesem Markt. Ihr Interesse ist es, das jeweilige Fach visibel zu machen und im Wettbewerb mit andern Fächern günstig zu positionieren. Fachgesellschaften können selbst als Verleger in Erscheinung treten (wie z. B. APA), zum Teil treten sie auch als Akteure im Rahmen von Open-Access-Aktivitäten in Erscheinung.

\section{Produkte und Rohstoffe}

Was sind die „Waren“, die auf diesem IuK-Markt gehandelt werden? Zum einen geht es natürlich um empirischexperimentelle Arbeiten - das also, was das Herzstück wissenschaftlicher Forschung bildet. Dieser Rohstoff, der von Wissenschaftlern erzeugt wird, erhält seine Politur durch das beste Qualitätssicherungsverfahren, das man sich vorstellen kann: Peer Reviewing. Die gegenseitige Kontrolle von Wissenschaftlern durch Wissenschaftler ist sicher einer der mächtigsten Faktoren zur Qualitätssicherung. Auch wenn dieses Verfahren bekannte Schwachstellen aufweist (z. B. Zitations- und GutachterKartelle), ist es gegenüber jeder anderen Möglichkeit der Qualitätskontrolle langfristig im Vorteil. Der veredelte Rohstoff wird in speziellen Publikationsorganen veröffentlicht, deren Wertigkeit durch den schon genannten Impact-Faktor charakterisiert wird.

Ein zweites Produkt sind Text- und Lehrbücher, in denen eine Vielzahl empirischer Befunde geordnet und zusammengetragen werden. Hier ist das Qualitätssicherungsverfahren vielfach reduziert bzw. in die Hand von Lektoren gelegt, deren wichtigste Kriterien Fragen der Marktgängigkeit und Verkaufbarkeit von solchen Texten betreffen. Allerdings sind durch moderne Produktionstechniken (,Book on Demand“) auch Vertriebsformen entstanden, die für kleine Auflagen kostengünstige Veröffentlichungsmöglichkeiten jenseits großer Verlage bieten. Davon wird jedoch derzeit noch wenig Gebrauch gemacht.

Ein Markt, in dem viel Geld zu verdienen ist, betrifft Messinstrumente und Testverfahren. Hierfür haben sich besondere Strukturen (Firmen und Verlage) gebildet, die Herstellung und Vertrieb der entsprechenden Gerätschaften und Materialien übernehmen. Durch die Einführung von DIN-Normen (Westhoff et al., 2004) wird auch in diesem Bereich zunehmend auf Qualitätskontrolle und Qualitätssicherung Wert gelegt.

Neben diesen Hauptprodukten sind auch eine Reihe weiterer „Rohstoffe“ auf dem IuK-Markt zu handeln. Hierzu zählen etwa Rohdaten (z. B. PsychData oder Zentralarchiv für empirische Sozialforschung) oder AdressSammlungen (z. B. der inzwischen kostenpflichtige „Psychologen-Kalender“"im Internet).

\section{Kriterien und Zieldimensionen}

Die vorliegende Gemengelage ist insofern als komplex zu bezeichnen, als eine Vielzahl zum Teil konfligierender Ziele 
und Kriterien beteiligt sind. $\mathrm{Zu}$ diesen gehören etwa die Visibilität von Autoren, die Kosten der Produktion wie der Rezeption von Publikationen, Fragen von Informationsfreiheit und Informationspflicht, Fragen des Urheberrechts sowie Fragen, die etwa die Repräsentanz eines Faches im Spektrum der wissenschaftlichen Disziplinen oder die Repräsentanz einer Nation im weltweiten Wissenschaftsbetrieb betreffen. Die Vielfalt dieser verschiedenen „Spieler" und die dadurch notwendigerweise bedingten Vielfalt von Zielkriterien macht eine Beurteilung der Situation und eine Bewertung möglicher Alternativen fast unmöglich und kann immer nur bestimmte Interessenlagen berücksichtigen. Andererseits sehen wir die Situation von der Zielrichtung her als ganz eindeutig: Die Produkte der Wissenschaft sind öffentliches Gut und müssen daher frei verfügbar sein. Finanziell profitierende Dritte (z. B. Verlage) haben kein Recht auf die Bestandssicherung überflüssig gewordener Dienste durch nicht mehr notwendige indirekte öffentliche Subventionen, sondern sind aufgefordert, sich durch neue Dienste in der veränderten Landschaft zu etablieren.

\section{Empfehlungen und Lösungsvorschläge}

Wie stehen andere psychologische Gesellschaften zum Thema des Open Access? In der American Psychological Association (APA) wird eine ,,sowohl - als auch“ Bewertung gegeben. Einerseits wird Open Access grundsätzlich begrüßt. Andererseits wird auf mögliche Kosten verwiesen, die mit einem Open Access-Verfahren verbunden sind. Um die Qualität der wissenschaftlichen Veröffentlichungen hoch zu halten, sind die Regeln der qualitativen Sicherung wissenschaftlicher Arbeiten (Peer Review-Verfahren) einzuhalten. Darüber hinaus entstehen Kosten durch die Gestaltung und Formatierung der Texte, die standardisierten Vorgaben entsprechen müssen. Gegenwärtig werden die wahren Kosten für einen wissenschaftlichen Artikel auf \$ 3,600-8,000 geschätzt. Wenn dieser Wert auch relativ hoch gegriffen ist, kann vermutet werden, dass -3,000 eine realistische Schätzung der Kosten darstellt.

Nur langsam reagiert die Gesetzgebung auf die zunehmende digitale (immaterielle) Verbreitung geistigen Eigentums und tut sich schwer mit einer angemessenen Berücksichtigung der Interessen der Wissenschaft. In den USA wird dazu ein Public Access to Science Act diskutiert. In Deutschland arbeitet man zur Zeit am sog. ,Zweiten Korb“ der Urheberrechtsnovellierung und wird auf Grund neuer Vorgaben des Rats der Europäischen Union wohl gar einen „dritten Korb“ brauchen. Gelöst werden muss u. a. die beim ,ersten Korb“ zurückgestellte Frage, ob und unter welchen Bedingungen Dritte digitale Kopien anfertigen dürfen (z. B. Bibliotheken für die Fernleihe).

Wir sind uns einig, dass die Kosten, die durch OnlinePublikation entstehen, nicht einseitig durch die Autoren getragen werden können, zumindest wenn sie nicht mit öffentlichen Mitteln substituiert werden. Ein entsprechendes Modell würde sich als Hemmschuh für weitere wis- senschaftliche Publikationen erweisen. Alternative Vorschläge gehen in die Richtung, auf der Grundlage des bestehenden Publikationssystems Komponenten des Open Access zu installieren, die dazu führen, dass wissenschaftliche Inhalte zu niedrigen Kosten zur Verfügung gestellt werden. Konsequenterweise können wissenschaftliche Institutionen ihre Bibliotheksmittel (Ausgaben für Zeitschriftenabos) in die Publikationstätigkeit ihrer Autoren umleiten. Die Publikation von Forschungsergebnissen würde ähnlich der „Publikation“ in Form von Kongressbeiträgen als Teil der Forschungstätigkeit budgetiert. Ein weiteres Anliegen besteht darin, Mehrwert zu schaffen, indem bestehende Publikationsformate mit neuen Möglichkeiten, die sich durch das Internet ergeben, verbunden werden. Dabei ist auch zu berücksichtigen, dass eine Qualitätskontrolle stattfindet. Diese umfasst die Sicherung der Unverfälschtheit der Informationen, die durch geeignete Authentifizierungs- und Schutzmechanismen (z. B. digitales Wasserzeichen) garantiert wird.

Ein weiterer wichtiger Gesichtspunkt ist die Berücksichtigung der Präferenzen der Autoren. Dazu führen wir eine Internet-basierte Befragung von DGPs-Mitgliedern durch, die deren Präferenzen verdeutlichen wird. Generell ist es wichtig, die Qualität wissenschaftlicher Veröffentlichungen zu erhalten. Diesem Ziel dient ein dezentrales System, so dass eine zentrale Steuerung wissenschaftlicher Veröffentlichungen durch designierte Institutionen problematisch erscheint. Die Stärke der Wissenschaft resultiert nicht zuletzt aus ihrer dezentralen Organisationsstruktur.

Wir schlagen vor, dass die DGPs der ,Berliner Erklärung über Offenen Zugang zu Wissenschaftlichem Wissen" beitritt ${ }^{2}$. Damit sind die Grundlagen geschaffen, um die Vorteile einer Open Access-Initiative für die Psychologie als Wissenschaft zu sichern. Zwar gibt es in diesem Bereich unterschiedliche Interessen (wie die der Wissenschaftler, der Autoren, der Universitätsinstitute, der Bibliotheken und der Verlage), die miteinander in Übereinstimmung gebracht werden müssen, aber eine Orientierung an den Grundlagen von Open Access ist unstrittig.

\section{Perspektiven}

Welche Perspektiven lassen sich für die Informationsgesellschaft in den nächsten Jahren erwarten? Die Bedeutung der selbstgesteuerten Publikationen wird zunehmen. Autoren und Herausgeberteams sind in der Lage, druckreife Manuskripte zu erstellen. Das gilt selbst in den Disziplinen, in denen die Erstellung des Druckbildes durch komplizierte Formeln erschwert ist. Die Entwicklung spezieller e-Publishing-Systeme ermöglicht es, qualitativ hochwertige Formate selbst herzustellen. Die Zukunft wird

\footnotetext{
2 Auf der Mitgliederversammlung der DGPs am 28. 9. 2004 erfolgt mit der Anregung, Konsequenzen zu diskutieren und Publikationsmodelle $\mathrm{zu}$ diskutieren und Publikationsmodelle $\mathrm{zu}$ prüfen. Dies erfolgt gegenwärtig.
} 
durch eine größere Selbstkontrolle über die Erstellung eines druckreifen Manuskripts gekennzeichnet sein, das im Internet zur Verfügung gestellt werden kann.

\section{Abschluss}

Das Internet berührt und verändert psychologische Tätigkeit in vielfältiger Weise. Internet-basierte Forschung hat sich etabliert und bereichert das Methodenspektrum. Die Einführung dieser Forschungsmethodik in die Ausbildung von Psychologinnen und Psychologen wird empfohlen. Neue Kommunikationsformen entstehen durch die vielfältigen Dienste, die im Internet angeboten werden. Informationssuche gestaltet sich einerseits vielfach einfacher und mächtiger, andererseits entstehen neue Probleme in der Handhabung und beim Zugang zu den Informationen. Bei der Open-Access-Initiative handelt es sich um den Versuch, wissenschaftliche Publikationen der Fachöffentlichkeit zugänglich zu machen, ohne dass eine Kostenbarriere für den Nutzer gegeben ist. Wie in der „Berliner Erklärung über Offenen Zugang zu wissenschaftlichem Wissen" betont wird, hat das Internet eine große Bedeutung für die Verbreitung von wissenschaftlichem Wissen, da die Möglichkeit besteht, eine umfassende Repräsentation dieses Wissens der interessierten Öffentlichkeit auf der Basis eines schnellen Zugriffs zur Verfügung zu stellen.

Das Internet ist ein Medium zur Wissensverbreitung, das das wissenschaftliche Publizieren schon verändert hat und langfristig weiter verändern wird. Besonders wichtig ist in diesem Zusammenhang, dass wissenschaftliche Informationen breit gestreut und den wissenschaftlich arbeitenden Experten leicht zugänglich gemacht werden. Daher geht es um einen offenen Zugang zu relevanten Informationen über das Internet. Dieser wird durch das Prinzip des Open Access verwirklicht. Damit verbunden ist die Vision einer umfassenden und allgemein zugänglichen Wissensrepräsentation. Beiträge nach dem Prinzip des offenen Zugangs umfassen sowohl wissenschaftliche Forschungsergebnisse als auch weitere qualitative Daten und Materialien, die wissenschaftlich genutzt werden können. In diesem Sinne ist es das Ziel der „Berliner Erklärung über Offenen Zugang zu wissenschaftlichem Wissen", dass die Möglichkeiten des Internet genutzt werden, um die Transparenz und Verfügbarkeit wissenschaftlichen Wissens zu vergrößern.

\section{Literatur}

Birnbaum, M. H. (2004). Human research and data collection via the Internet. Annual Review of Psychology, 55, 803-832.

Birnbaum, M. H. \& Reips, U.-D. (in press). Behavioral research and data collection via the Internet. In R. W. Proctor \& K.-P. L. Vu (Eds.), The handbook of human factors in Web design. Mahwah, NJ: Erlbaum.

Bosnjak, M. (2003). Web-basierte Fragebogenuntersuchungen. Methodische Möglichkeiten, aktuelle Themen und Erweiterungen. In Informationszentrum Sozialwissenschaften (IZ)
\& Arbeitskreis Sozialwissenschaftlicher Institute e. V. (ASI) (Hrsg.), Online-Erhebungen (S. 109-133). Bonn: IZ.

Buchanan, T. (2001). Online personality assessment. In U.-D. Reips \& M. Bosnjak (Eds.), Dimensions of Internet Science (pp. 57-74). Lengerich: Pabst.

Buchanan, T. (2002). Online assessment: Desirable or dangerous? Professional Psychology: Research and Practice, 33, $148-154$.

Eichstaedt, J. (2001). Reaction time measurement by JAVAapplets implementing Internet-based experiments. Behavior Research Methods, Instruments, and Computers, 33, 179186.

Funke, J., Stumpf, M., Weichselgartner, E. \& Wilkening, F. (2003). Qualitätssicherung im Bereich neuer Medien durch Einführung von Qualitätskriterien. In R. Ott \& C. Eichenberg (Hrsg.), Klinische Psychologie und Internet. Potenziale für klinische Praxis, Intervention, Psychotherapie und Forschung (S. 99-113). Göttingen: Hogrefe.

Harnad, S. (1990). Scholarly sky-writing and the prepublication continuum of scientific inquiry. Psychological Science, 1, 342-344.

Joinsen, A. N. (2003), Understanding the psychology of Internet behavior: Virtual worlds, real lives. Basingstoke: Palgrave Macmillan.

Krantz, J. H. \& Dalal, R. (2000). Validity of Web-based psychological research. In M. H. Birnbaum (Ed.), Psychological Experiments on the Internet (pp. 35-60). San Diego, CA: Academic Press.

Musch, J. \& Reips, U.-D. (2000). A brief history of Web experimenting. In M. H. Birnbaum (Ed.), Psychological experiments on the Internet (pp. 61-88). San Diego, CA: Academic Press.

Reips, U.-D. (2000). The Web Experiment Method: Advantages, disadvantages, and solutions. In M. H. Birnbaum (Ed.), Psychological experiments on the Internet (pp. 89118). San Diego, CA: Academic Press.

Reips, U.-D. (2002 a). Internet-based psychological experimenting: Five dos and five don'ts. Social Science Computer Review, 20 (3), 241-249.

Reips, U.-D. (2002 b). Standards for Internet-based experimenting. Experimental Psychology, 49, 243-256.

Reips, U.-D. (2003). Psychologische Forschung zum und im Internet. Psychologie in Österreich, 22 (1), 19-25.

Reips, U.-D. (in press). Web-based methods. In M. Eid \& E. Diener (Eds.), Handbook of psychological assessment: A multimethod perspective. Washington, DC: American Psychological Association.

Reips, U.-D., Morger, V. \& Meier B. (2001). „Fünfe gerade sein lassen": Listenkontexteffekte beim Kategorisieren. URL http://www.psychologie.unizh.ch/sowi/reips/papers/re mo_me2001.pdf

Westhoff, K., Hellfritsch, L. J., Hornke, L. F., Kubinger, K. D., Lang, F., Moosbrugger, H., et al. (Hrsg). (2004). Grundwissen für die berufsbezogene Eignungsbeurteilung nach DIN 33430. Lengerich: Pabst.

\section{Kommentierte URLs}

http://www.soros.org/openaccess/g/read.shtml

Die Budapest Open Access Initiative vom 17. 1. 2002

http://cogprints.ecs.soton.ac.uk/view/subjects/psyc.html

Das Selbstarchivierungsbeispiel „CogPrint" mit freiem VolltextZugang zu Artikeln aus der Psychologie

http://www.mpg.de/pdf/openaccess/BerlinDeclaration dt.pdf

Berliner Erklärung „Open Access to Knowledge in the Sciences and Humanities" vom Oktober 2003

http://www.publiclibraryofscience.org/

Einstiegsseite der „Public Library of Science“ (PloS)

http://www.hrk.de/presse/2821.htm 
Hochschul-Rektoren-Konferenz 5. 11. 2002: Zur Neuausrichtung des Informations- und Publikationssystems der deutschen Hochschulen

http://www.wissenschaftsrat.de/presse/pm_1601.htm

Wissenschaftsrat 16.7.2001: Empfehlungen zur digitalen Informationsversorgung durch Hochschulbibliotheken

http://www.ncbi.nlm.nih.gov/pubmed

Einstiegsseite in Medline/PubMed

http://www.isiwebofknowledge.com/

Homepage des ISI (Web of Science)

http://www.library.cornell.edu/scholarlycomm/problem.html

Beispielhafte Angaben der Cornell University (Stand 12/2003)

über den Anstieg der Zeitschriften-Preise für Universitätsbiblio-

theken

http://www.doaj.org/

Verzeichnis von Open Access Zeitschriften

http://www.iuk-initiative.org/documents/digbib05032002/

Positionspapier „Digitale Bibliotheken“ der Initiative Information und Kommunikation der wissenschaftlichen Fachgesellschaften in Deutschland

http://www.dl-forum.del

Förderaktivitäten und weitere Informationen rund um das Thema DIGITALE BIBLIOTHEK von BMBF und DFG

http://www.gutenberg.net/

Projekt Gutenberg (erste und größte Sammlung kostenloser elektronischer Volltexte); deutsche Ausgabe unter http://gutenberg. spiegel.del

http://www.psychologie.unizh.ch/sowi/Ulf/Lab/WebExpPsyLab. html

Web-Labor für Experimentelle Psychologie (1995 in Tübingen gegründet, ältestes Labor für Web-Experimente)

http://genpsylab-wexlist.unizh.ch/

Web experiment list (weltweit größte Liste aktiver und archivierter deutsch- und englischsprachiger Web-Experimente)

http://genpsylab-wexlist.unizh.ch/browse.cfm?action= browse \&modus $=$ survey
Web survey list (Liste aktiver und archivierter deutsch- und englischsprachiger Web-Befragungen)

http://psych.hanover.edu/research/exponnet.html

Psychological Research on the Net (John Krantz)

http://ipip.ori.org/ipip/

International Personality Item Pool (Lewis R. Goldberg)

http://www.socialpsychology.org/expts.htm

Online Social Psychology Studies (Scott Plous)

http://psych.fullerton.edu/mbirnbaum/programs/surveyWiz.htm SurveyWiz (von Michael Birnbaum. Eine Webseite zum Erstellen einfacher Befragungen)

http://www.inquiry.de/

Inquiry (FH Ravensburg-Weingarten, Befragungen)

http://psych-wextor.unizh.ch/wextor/en/index.php

WEXTOR (von Ulf-Dietrich Reips \& Christoph Neuhaus, Universität Zürich. Generieren, Visualisieren und Verwalten von experimentellen Versuchsdesigns, Erstellen von Webseiten und Versuchssteuerung)

http://www.psychologie.unizh.ch/sowi/reips/stroop/

Stroop Invaders (von Ulf-Dietrich Reips \& Beat Mürner, Universität Zürich. Erstellen von Stroop-Experimenten in Spiel-Format)

http://genpsylab-logcrunsh.unizh.ch/

Scientific LogAnalyzer (von Ulf-Dietrich Reips, Universität Zürich \& Stefan Stieger, Universität Wien. Auswertung beliebiger Logdateien)

Prof. Dr. Joachim Funke

Psychologisches Institut

Universität Heidelberg

Hauptstraße 47-51

69117 Heidelberg

E-Mail: joachim.funke@psychologie.uni-heidelberg.de 